\title{
HARRISON'S WITT RING OF A GENERALIZED
}

\section{VALUATION RING}

\author{
CHAN-NAN CHANG
}

\begin{abstract}
The purpose of this paper is to determine the relations between $H(R)$ (Harrison's Witt ring given by generators and relations over a generalized valuation ring $R$ ) and $W(K)$ (classical Witt ring of inner product spaces over the field $K$ ). Using the main results, we are able to establish some theorems on the structure of $H(R)$ analogous to the field case.
\end{abstract}

1. Preliminaries. For a commutative ring $R$, Harris on's Witt ring, $H(R)$, is the ring given by generators $\langle a\rangle, a \in R$, and relations

(H1) $\langle 0\rangle=0$,

(H2) $\langle a b\rangle=\langle a\rangle\langle b\rangle$, and

(H3) $\langle a\rangle+\langle b\rangle=\langle a+b\rangle+\langle(a+b) a b\rangle$.

It is shown in [3] and [4] that for a field $F, H(F)$ is the classical Witt ring of $F, W(F)$, based on inner product spaces over $F$.

An $H$-map is a mapping $t: R \rightarrow S$ of commutative rings satisfying $t(0)=$ $0, t(a b)=t(a) t(b)$ and $t(a)+t(b)=t(a+b)+t((a+b) a b)$. Thus \langle\rangle$_{R}($ or \langle\rangle$)$ : $R \rightarrow H(R)$ is a universal $H$-map.

$H$ is a functor from the category of commutative rings to the same category, where for a ring homomorphism $f: R \rightarrow S, H(f): H(R) \rightarrow H(S)$ is the unique ring homomorphism such that \langle\rangle$_{S} \cdot f=H(f) \cdot\langle\rangle_{R} \cdot$

1.1 Lemma. An element $r$ in $R$ is a unit if and only if $\left\langle r^{2}\right\rangle=1$ in $H(r)$.

Proof. Apply Lemmas 2.1, 2.3 and 3.1 in [1].

Let $L_{R}$ (or $L$ ) denote the ideal of $R$ generated by $x^{2} y+x y^{2}$ for all $x, y$ in $R$.

1.2 Lemma. If $L$ contains a unit, then $\langle r\rangle=\left\langle r^{3}\right\rangle$ for all $r$ in $R$.

Received by the editors November 12, 1973.

AMS (MOS) subject classifications (1970). Primary 10C05, 10E 25; Secondary 10C30, $20 \mathrm{G} 25$.

Key words and phrases. Valuation ring, Witt ring, Harrison's Witt ring, H-map, unimodular element, Jordan decomposition. 
Proof. Apply Lemmas 3.1 and 3.4 in [1].

2. Jordan decomposition of an element in $H(R)$. From now on we assume that $R$ is a generalized valuation ring, i.e. $R$ is a commutative, noetherian ring with identity whose ideals are totally ordered with respect to inclusion. Choose a prime $\pi$ in $R$ and fix it once and for all. Every ideal of $R$ is of the type $\pi^{n} R$ for some nonnegative integer $n$. Let $k_{R}$ (or $k$ ) denote the residue class field of $R$ (i.e. $k \simeq R / \pi R$ ). If $R$ is an integral domain, we denote the quotient field of $R$ by $F_{R}$ (or $F$ ). Let $-: R \rightarrow F$ be the canonical maps. We use - and $i$ to denote the induced maps $H(-)$ and $H(i)$, respectively.

2.1 Theorem. Suppose $R$ is not an integral domain. Then

$$
H(R) \simeq \begin{cases}Z_{2} \times Z_{2} & \text { if } k=Z_{2}, \\ W(k) & \text { if } k \neq Z_{2} .\end{cases}
$$

Proof. Suppose $k=Z_{2}$. Let $Z_{2}\left(C_{2}\right)$ denote the group ring of the group $C_{2}=\{1, x\}$ over $Z_{2}$. Define the map $t: R \rightarrow Z_{2}\left(C_{2}\right)$ as follows:

$$
t(r)= \begin{cases}1 & \text { if } r \in R, \\ x & \text { if } r \in \pi R \backslash \pi^{2} R, \\ 0 & \text { otherwise. }\end{cases}
$$

It is easy to check that $t$ is an $H$-map, and the homomorphism induced by $t$ is an isomorphism, so $H(R) \simeq Z_{2}\left(C_{2}\right)$.

Suppose $k \neq Z_{2}$. The radical of $R$ equals $\pi R$. Since $L$ contains a unit, by 1.2 and $[1,2.1],\langle\pi\rangle=0$. It follows from $[1,3.1]$ that $W(k) \simeq H(R / \pi R) \simeq$ $H(R) /\langle\pi\rangle H(R) \simeq H(R)$.

Theorem 2.1 determines $H(R)$ completely when $R$ is not an integral domain. So for the remainder of this paper we assume that $R$ is an integral domain.

Let $\dot{R}$ be the multiplicative group of units of $R$. We denote $\left\langle a_{1}\right\rangle+\cdots+$ $\left\langle a_{n}\right\rangle$ by $\left\langle a_{1}, \cdots, a_{n}\right\rangle$. An element $V$ in $H(R)$ is called unimodular provided $V=0$, or there exists $a_{1}, \cdots, a_{n} \in \dot{R}$ such that $V=\left\langle a_{1}, \cdots, a_{n}\right\rangle$, and if $b_{1}, \cdots, b_{n} \in R$ with $\Sigma a_{i} b_{i}^{2} \in \pi R$, then $b_{i} \in \pi R$ for all $i=1, \cdots, n$. Since

$$
\left(a_{1}+a_{2}\right) b_{1}^{2}+\left(a_{1}+a_{2}\right) a_{1} a_{2} b_{2}^{2}=a_{1}\left(b_{1}+a_{2} b_{2}\right)^{2}+a_{2}\left(b_{1}-a_{1} b_{2}\right)^{2},
$$

$\left\langle a_{1}, a_{2}\right\rangle$ is unimodular if and only if $\left\langle 1, a_{1} a_{2}\right\rangle\left\langle a_{1}+a_{2}\right\rangle$ is unimodular. Hence unimodularity is well defined. 
Let $I_{R}$ (or $I$ ) be the ideal of $H(R)$ generated by $\langle 1,-1+a \pi\rangle$ for all $a \in R$. Let $j_{r}$ (or $j$ ): $I_{R} \rightarrow H(R)$ be the canonical embedding.

2.2 Lemma. $\langle\pi\rangle=\left\langle\pi^{3}\right\rangle$ if and only if $k \neq Z_{2}$.

Proof. Sufficiency follows from 1.2. Conversely if $k=Z_{2}$ then $H(R) /\langle\pi\rangle H(R) \simeq H(k) \simeq W(k) \simeq Z_{2}$. On the other hand $H(R) /\left\langle\pi^{3}\right\rangle H(R) \simeq$ $H\left(R / \pi^{3} R\right) \simeq Z_{2} \times Z_{2}$ by 2.1 . Hence $\langle\pi\rangle \neq\left\langle\pi^{3}\right\rangle$.

2.3 Lemma. Suppose $V$ is a unimodular element in $H(R)$. If $V \in I$ then $V=0$.

Proof. It follows directly from definitions.

We let $s=2$ if $k \neq Z_{2}$ and $s=3$ if $k=Z_{2}$.

2.4 Proposition. Let $V \in H(R)$. Then there exist unimodular elements $V_{0}, V_{1}, \cdots, V_{s} \in H(R)$ and $V_{s+1} \in I$ such that

$$
V=V_{0}+\langle\pi\rangle V_{1}+\cdots+\left\langle\pi^{s}\right\rangle V_{s}+V_{s+1} .
$$

Proof. Let $V=\left\langle a_{1}, \cdots, a_{n}\right\rangle$ with $a_{i} \in R$ for $i=1, \cdots, n$. We use induction on $n$. If $n=1$ it follows from 1.2 and $[1,2.1]$. We assume $n>1$. Let $\left\langle a_{1}, \cdots, a_{n-1}\right\rangle=U_{0}+\cdots+\left\langle\pi^{s}\right\rangle U_{s}+U_{s+1}$, where $U_{0}, \cdots, U_{s}$ are unimodular elements and $U_{s+1} \in I$. By 1.2 and $[1,2.1], a_{n}$ can be chosen to be $\pi^{i} b$, where $b \in R$ and $0 \leq i \leq s$. Let $U_{i}=\left\langle b_{1}, \cdots, b_{m}\right\rangle$ where $b_{1}, \cdots, b_{m}$ are units. If $U_{i}+\langle b\rangle$ is unimodular, then it is done. If $U_{i}+\langle b\rangle$ is not unimodular there exist units $c_{j_{1}}, \cdots, c_{j_{l}}$, with $1 \leq l \leq m$, such that $b_{j_{1}} c_{j_{1}}^{2}+\cdots+b_{j_{l}} c_{j_{l}}^{2} \equiv-b(\bmod \pi R)$. By using $b_{j_{a}} c_{j_{a}}^{2}$ instead of $b_{j_{a}}, a=$ $1, \cdots, l$, and by rearranging the $b_{j_{a}}$ 's, we may assume, without loss of generality, that $b_{1}+\cdots+b_{l} \equiv-b(\bmod \pi R)$. Hence $U_{i}+\langle b\rangle=U_{i}^{\prime}+$ $\left\langle b_{1}+\cdots+b_{l}, b\right\rangle$, where

$$
U_{i}^{\prime}=\sum_{j=2}^{l}\left\langle\left(b_{1}+\cdots+b_{j-1}\right) b_{j}\left(b_{1}+\cdots+b_{j}\right)\right\rangle+\left\langle b_{l+1}, \cdots, b_{m}\right\rangle .
$$

We only have to show that $U_{i}^{\prime}$ is unimodular. Let $b_{j}^{\prime}=\left(b_{1}+\cdots+b_{j-1}\right)$ $\cdot b_{j}\left(b_{1}+\cdots+b_{j}\right)$ for $j=2, \cdots, l$, and $b_{j}^{\prime}=b_{j}$ for $j=l+1, \cdots, m$. Let $d=$ $\sum_{j=2}^{m} d_{j}^{2} b_{j}^{\prime}$ where $d_{j} \in R$. It follows from induction on $l$ and straightforward computations that 
$d=b_{1}\left(\sum_{j=2}^{l} d_{j} b_{j}\right)^{2}+\sum_{j=2}^{l} b_{j}\left(-d_{j}\left(b_{1}+\cdots+b_{j-1}\right)+\sum_{a=j+1}^{l} b_{\alpha} d_{\alpha}\right)^{2}+\sum_{j=l+1}^{m} b_{j} d_{j}^{2}$.

If $d \in \pi R$, then by the unimodularity of $U_{i}$,

$$
\sum_{a=j+1}^{l} b_{\alpha} d_{\alpha}-d_{j}\left(b_{1}+\cdots+b_{j-1}\right) \in \pi R
$$

for $j=2, \cdots, l$, and $d_{j} \in \pi R$ for $j=l+1, \cdots, m$. Solving the system of $(l-1)$ linear equations in $(l-1)$ unknowns, $d_{2}, \cdots, d_{l}$, it follows that $d_{i} \in \pi R$ for $i=2, \cdots, l$. Hence $U_{i}$ is unimodular.

For each $V \in H(R)$, the decomposition (2.1) of $V$ is called a Jordan decomposition of $V$. The decomposition depends on the choices of the prime element $\pi$ in $R$, and it is not even unique with respect to a fixed prime $\pi$. However we will show that the decomposition is unique modulo $I$.

2.5 Lemma. Let $V=\Sigma_{m=1}^{s}\left\langle\pi^{m}\right\rangle V_{m} \in H(R)$, where $V_{m}$ is unimodular for $m=1, \cdots, s$. Then $V \in I$ if and only if $V_{m}=0$ for $m=1, \cdots, s$.

Proof. Necessity follows from the unimodularity of $V_{m}$ and 2.3. Sufficiency is obvious.

2.6 Theorem. Let $V \in H(R)$ and let

$$
V=\sum_{m=0}^{s}\left\langle\pi^{m}\right\rangle V_{m}+V_{s+1}=\sum_{m=0}^{s}\left\langle\pi^{m}\right\rangle U_{m}+U_{s+1}
$$

be two Jordan decompositions of $V$. Then $\bar{V}_{m}=\bar{U}_{m}$ in $W(k)$ for $m=0, \cdots, s$.

Proof. It is easy to see that $\bar{V}_{0}=\bar{V}=\bar{U}_{0}$ in $H(k)=W(k)$. Thus $V_{0}-$ $U_{0} \in I$ and $\Sigma_{m=1}^{s}\left\langle\pi^{m}\right\rangle\left(V_{m}-U_{m}\right) \in I$. Since $V_{m}-U_{m}$ is of the form $\Sigma\left\langle u_{j}\right\rangle$, $u_{j}$ units, it can be written as $W_{0}+W_{1}$, where $W_{0}$ is unimodular and $W_{1} \in I$. Then apply 2.5 .

3. Associate $H(R)$ with $W(k)$ and $W(F)$. Let $V \in H(R)$ with Jordan decomposition (2.1). Define a map $\rho: H(R) \rightarrow \bigoplus_{m=0}^{s} W(k)$ by letting $\rho(V)=$ $\left(\bar{V}_{0}, \cdots, \bar{V}_{s}\right)$. By $2.5, \rho$ is well defined.

Let $V=\Sigma\left\langle a_{m}\right\rangle\left\langle 1,-1+b_{m}\right\rangle \in I$. Define a map $\eta: I \rightarrow W(F)$ by letting $\eta(V)=\Sigma\left\langle a_{m}\right\rangle_{F}\left\langle 1,-1+v_{m}\right\rangle_{F} \in W(F)$.

In this section, we will show that $\operatorname{ker} \rho=I$ and $\eta$ is injective. By using these results, we prove that $H_{t}(R)$, the torsion subgroup of $H(R)$, is 2-primary. 
3.1 Proposition. $\rho$ is a surjective additive homomorphism with kernel $I$.

Proof. It is easy to see that $\rho$ is a surjective additive homomorphism and $\operatorname{ker} \rho \supseteq I$. ker $\rho \subseteq I$ follows from 2.3 and 2.5 .

3.2 Corollary. If $R$ lifts squares (i.e. $1+\alpha \pi$ is a square for $\alpha \in R$ ), then $I=\{0\}$ and $H(R) \simeq \bigoplus_{m=0}^{s} W(k)$.

3.3 Proposition. If $D$ is a Dedekind domain with quotient field $K$, then $H(D) \rightarrow H(K) \times \pi H\left(D / P^{2}\right)$ is one-one, where $P$ ranges over all nonzero prime ideals of $D$.

Proof. See [2, Corollary 1.6].

3.4 Theorem. $\eta$ is an injective ring homomorphism.

Proof. $\operatorname{Ker}\left(H(R) \rightarrow H\left(R / \pi^{2} R\right)\right)=\left\langle\pi^{2}\right\rangle H(R) \supseteq\left\langle\pi^{2}\right\rangle I_{R}=I_{R}$. By 3.3,

$$
\operatorname{Ker}\left(H(R) \rightarrow H\left(R / \pi^{2} R\right)\right) \cap \operatorname{Ker}(H(i))=0 .
$$

Thus $0=I_{R} \cap \operatorname{Ker} H(i) \supseteq I_{R} \cap \operatorname{Ker} \eta$, so $\eta$ is injective.

3.5 Theorem. The torsion subgroup of $H(R), H_{t}(R)$, is a 2-primary subgroup.

Proof. Let $K$ be a field. Then $W_{t}(K)$, the torsion subgroup of $W(K)$, is 2-primary. Let $V=\Sigma_{m=0}^{s}\left\langle\pi^{m}\right\rangle V_{m}+V_{s+1} \in H_{t}(R)$. Then each $\bar{V}_{m} \in W_{t}(k)$ for $m=0, \cdots, s$. By 3.1 and 3.4 there exists some nonnegative integer $n$

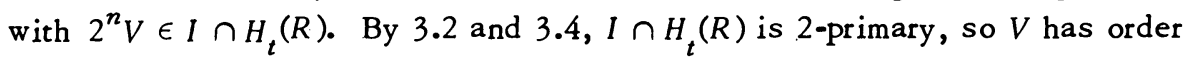
a power of 2 .

4. Transfer maps. Let $R$ and $S$ be two generalized valuation domains with $R$ a subring of $S$. Let $f: R \rightarrow S$ be the canonical embedding. In this section, we try to find the kernel of $H(f)$ in certain special cases.

The stufe of $R, s(R)$, is the smallest integer $m$ such that $-1=a_{1}^{2}+\cdots+$ $a_{m}^{2}$ with $a_{i} \in R$ for $i=1, \cdots, m$. It has been shown in [5] that $s$ is a power of 2 .

A ring is called pythagorean if every sum of squares is a square itself. The pythagorean closure of a ring $R, R_{p}$, is the minimal pythagorean overring of $R$. If $R$ is a generalized valuation domain, then $R_{p}$ can be obtained by adjoining elements of the form $1+a^{2}$ for $a \in R$.

4.1 Proposition. Suppose $R, S$ are fields. If $S / R$ is a finite extension of odd degree, then $H(f)$ is injective. 
Proof. The same proof as that of [7, Theorem 2.2.2] works for arbitrary fields $R$ and $S$.

4.2 The orem. Suppose that $S / R$ is a totally ramified extension when $k_{R}=Z_{2}$. If the rank of $S$ over $R$ is odd then $H(f)$ is injective.

Proof. It is easy to check that the following diagram commutes and the rows are exact by 3.1 .

$$
\begin{aligned}
0 & \rightarrow I_{R} \rightarrow H(R) \rightarrow \bigoplus_{m=0}^{s} W\left(k_{R}\right) \rightarrow 0 \\
& \left\lfloor W\left(f_{F}\right)\lfloor H(f)\rfloor \bigoplus W\left(f_{k}\right)\right. \\
0 & \rightarrow I_{S} \rightarrow H(s) \rightarrow \bigoplus_{m=0}^{s} W\left(k_{s}\right) \rightarrow 0
\end{aligned}
$$

where $\bigoplus W\left(f_{k}\right)$ is well defined from the assumptions. By 4.1 and 3.4, $W\left(f_{F}\right)$ and $\bigoplus W\left(f_{k}\right)$ are injective. Hence $H(f)$ is injective.

4.3 Theorem. Suppose $k_{R}=Z_{2}$ and $S / R$ is an extension of odd degree over $R$ which is not totally ramified. Then $\operatorname{Ker} H(f)=\left\langle\pi,-\pi^{3}\right\rangle H(R)$.

Proof. It follows from the assumption that $k_{S} \neq Z_{2}$, thus $\left\langle\pi,-\pi^{3}\right\rangle_{S}=0$ by 1.2. This shows $\left\langle\pi,-\pi^{3}\right\rangle H(R) \subseteq \operatorname{Ker} H(f)$. Conversely, we define a map $g: \bigoplus_{m=0}^{3} W\left(k_{R}\right) \rightarrow \bigoplus_{m=0}^{2} W\left(k_{S}\right)$ by letting

$$
g\left(\bar{V}_{0}, \cdots, \bar{V}_{3}\right)=\left(\bar{V}_{0}, \bar{V}_{1}+\bar{V}_{3}, \bar{V}_{2}\right) \in \bigoplus_{m=0}^{2} W\left(k_{s}\right)
$$

for every $\left(\bar{V}_{0}, \cdots, \bar{V}_{3}\right)$ in $\bigoplus_{m=0}^{3} W\left(k_{R}\right)$. By 4.1 and $[1,2.1,2.3], g$ is a welldefined homomorphism with $\operatorname{ker} g=\left\{(0, \bar{V}, 0, \bar{V}) \mid \bar{V} \in W\left(k_{R}\right)\right\}$. Use $g$ instead of $\bigoplus W\left(f_{k}\right)$ in (4.1); then (4.1) still commutes since $\langle\pi\rangle=\left\langle\pi^{3}\right\rangle$ in $H(S)$. By 4.1 and diagram chasing, $\left\langle\pi,-\pi^{3}\right\rangle H(R)^{\prime} \supseteq \operatorname{Ker} H(f)$.

4.4 Lemma. Let $S=R(\theta)$, where $\theta$ satisfies $x^{2}-a x-1=0$ for some $a \in R$. Then $\operatorname{Ker}(H(f))$ is a torsion subgroup of $H(R)$.

Proof. Suppose $R$ is a field. If char $R=2$ then $H(R)=H_{t}(R)$; we are done. If char $R \neq 2$, then the lemma is Theorem 2.2.3 in [7].

In general, let $V \in \operatorname{Ker}(H(f))$. If char $k_{R}=2$, then $\rho_{R}(V) \in \bigoplus_{m=0}^{s}{ }_{t}\left(k_{R}\right)$ is obvious. If char $k_{R} \neq 2$, then it follows from 4.1 and the above discussion that $\rho_{R}(V) \in \bigoplus_{m=0}^{2} W_{t}\left(k_{R}\right)$. Thus $n \rho_{R}(V)=0 \in \bigoplus_{m=0}^{s} W\left(k_{R}\right)$. By the exactness of (4.1), $n V \in I_{R} \cdot \eta_{S} \cdot w\left(f_{F}\right)(n V)=H(f)(n V)=0$. So $W\left(f_{F}\right)(n V)=0$ and $n V$ is torsion. 
4.5 Theorem. (a) If $s(R)<\infty$ then $H(R)$ is a torsion group.

(b) If $s(R)=\infty$ then $H_{t}(R)=\operatorname{ker}(H(f))$ where $f: R \rightarrow R_{p}$ is the canonical embedding.

Proof. If $s(R)<\infty$, then $s(F)$ and $s(k)$ are finite. Thus $W(F)$ and $W(k)$ are torsion. Apply 3.1 and 3.4.

Suppose $s(R)=\infty$. Let $V=\left\langle a_{1}, \cdots, a_{m}\right\rangle \in H_{t}(V) . H(f)(V)$ represents the same elements in $R$ as $n H(f) V$, i.e. the set $\left\{\Sigma a_{i} u_{i}^{2} \mid u_{i} \in R_{p}\right\}$ is the same as $\left\{\sum_{i=1}^{m} a_{i}\left(\sum_{j=1}^{n} u_{i j}^{2}\right) \mid u_{i j} \in R_{p}\right\}$. Hence $H(f)(V)=0$ by $(\mathrm{H} 3)$ and induction on $m$. Conversely, let $S=R(\theta)$ where $\theta=-a+\left(1+a^{2}\right)^{1 / 2}$ for $a \in R$. $\theta$ satisfies the equation $x^{2}+2 a x-1=0$. By 4.4, $\operatorname{Ker} H\left(f_{S / R}\right)$ is torsion. Now ker $H(f) \subseteq H_{t}(R)$ follows from induction.

\section{REFERENCES}

1. D. Coleman and J. Cunningham, Harrison's Witt ring of a commutative ring, J. Algebra 18 (1971), 549-564. MR $44 \# 1682$.

2. - Comparing Witt rings, J. Algebra 28 (1974), 296-304.

3. D. Coleman, Embedding Witt ring of Dedekind domains, J. Number Theory 6 (1974), 232-237.

4. D. Harrison, Witt rings, Lecture Notes, University of Kentucky, Lexington, Ky., 1970.

5. M. Knebusch, Runde Formen uber semilokalen Ringen, Math. Ann. 193 (1971), 21-34. MR $45 \# 172$.

6. J. Milnor, Symmetric inner product in characteristic 2, Prospects in Mathematics, Annal. Study 70, 1972, pp. 59-75.

7. W. Scharlau, Quadratic forms, Queens Papers in Pure and Appl. Math., no. 22, Queens University, Kingston, Ontario, 1969. MR 42 \#4574.

DEPARTMENT OF MATHEMATICS AND STATISTICS, UNIVERSITY OF MASSACHU• SETTS, AMHERST, MASSACHUSETTS 01002 